Portland State University

PDXScholar

2-21-2006

\title{
Similarity-Based Constitutive Relations for Local Mass Fluxes in Incompressible Mixing Layers
}

John D. Ramshaw

Portland State University, jdramshaw@yahoo.com

Follow this and additional works at: https://pdxscholar.library.pdx.edu/phy_fac

Part of the Physics Commons

Let us know how access to this document benefits you.

Citation Details

J. D. Ramshaw, "Similarity-based constitutive relations for local mass fluxes in incompressible mixing layers," Phys. Rev. E. 73, 026309 (2006)

This Article is brought to you for free and open access. It has been accepted for inclusion in Physics Faculty Publications and Presentations by an authorized administrator of PDXScholar. Please contact us if we can make this document more accessible: pdxscholar@pdx.edu. 


\title{
Similarity-based constitutive relations for local mass fluxes in incompressible mixing layers
}

\author{
John D. Ramshaw* \\ Boise, Idaho 83704, USA \\ (Received 27 August 2005; published 21 February 2006)
}

\begin{abstract}
The local concentrations of the two fluids within a mixing layer produced by an interfacial instability are determined by their individual continuity equations. Solution of these equations requires constitutive relations for the local mass fluxes of the two fluids. We derive explicit analytical expressions for these fluxes in planar incompressible mixing layers characterized by a single integral length scale $h(t)$, which is presumed to be provided by a suitable mix or turbulence model. Elementary scaling arguments imply that in mixing layers of this type, the mean volume fraction profile $\alpha(x, t)$ depends on $x$ and $t$ only through the similarity variable $s$ $=x / h(t)$. The continuity equations determine $\alpha(s)$ in terms of the mass fluxes. We invert this relationship to obtain constitutive relations for the mass fluxes in terms of an arbitrary given $\alpha(s)$ obtained from experiment or direct numerical simulation. These relations can then be employed in more complicated situations (e.g., problems lacking spatial symmetry) in which the volume fraction is not known a priori. The resulting mass fluxes are not inherently diffusional in character, but can be transformed into an alternative equivalent diffusional form that may be advantageous for computational purposes. These results are further generalized to the case in which the light and heavy regions of the mixing layer exhibit different piecewise self-similar scaling behavior characterized by two independent integral length scales $h_{1}(t)$ and $h_{2}(t)$ that depend differently on time. The validity and practical utility of the formulation are confirmed by a test calculation.
\end{abstract}

DOI: 10.1103/PhysRevE.73.026309

PACS number(s): 47.20.Ma

\section{INTRODUCTION}

There is a continuing lively interest in the mixing layers produced by the nonlinear growth of perturbations at unstable interfaces that separate a lighter fluid $A$ from a heavier fluid $B$. The simplest quantitative characterization of such mixing layers is in terms of some appropriate integral length scale $h(t)$ proportional to the width or thickness of the mixing layer at time $t$. The three classical interfacial instabilities are the well-known Rayleigh-Taylor (RT), RichtmyerMeshkov (RM), and Kelvin-Helmholtz (KH) instabilities, for which $h(t)$ obeys simple scaling laws [1-9]. In practical applications, however, these instabilities are rarely encountered in their idealized pure forms. In particular, the interfacial acceleration history is rarely constant (RT) or impulsive (RM), but usually exhibits a more complicated time dependence. The simple scaling laws are then no longer directly applicable, and it becomes necessary to construct dynamical models for the resulting time evolution of $h(t)$. The simplest such models take the form of ordinary differential equations (ODE's) for $h(t)$. Various authors have proposed various ODE mix models of this type [5-7,9-14], which tend to be similar in structure and to produce similar (and often remarkably good) agreement with experimental measurements of $h(t)$.

By their very nature, however, such ODE mix models cannot, and do not pretend to, predict or even describe the internal structure of the mixing layer. The most basic description of this structure is in terms of the ensemble averaged concentrations or partial mass densities $\rho_{A}$ and $\rho_{B}$ of the two fluids as functions of position and time. These mass

\footnotetext{
*Email address: john@ramshaw.org
}

densities are determined by the continuity equations of the two fluids, the solution of which in turn requires knowledge of the corresponding fluid velocity fields $\mathbf{u}_{A}$ and $\mathbf{u}_{B}$. The simplest fluid dynamical descriptions of this type are those in which a single momentum equation determines the massweighted mean velocity $\mathbf{u}$ of the mixture as a whole, which is related to $\mathbf{u}_{A}$ and $\mathbf{u}_{B}$ by

$$
\rho \mathbf{u}=\rho_{A} \mathbf{u}_{A}+\rho_{B} \mathbf{u}_{B},
$$

where $\rho=\rho_{A}+\rho_{B}$ is the total mass density of the mixture. In order to determine $\mathbf{u}_{A}$ and $\mathbf{u}_{B}$ separately, Eq. (1) must be supplemented by a second relation between these velocities, which is ordinarily obtained $[15,16]$ from a constitutive relation for the corresponding mass fluxes relative to $\mathbf{u}$. These fluxes are defined by

$$
\begin{gathered}
\mathbf{J}_{A}=\rho_{A}\left(\mathbf{u}_{A}-\mathbf{u}\right), \\
\mathbf{J}_{B}=\rho_{B}\left(\mathbf{u}_{B}-\mathbf{u}\right)=-\mathbf{J}_{A} .
\end{gathered}
$$

More elaborate two-fluid models, in which the velocity of each fluid is determined by its own momentum equation, have also been proposed (e.g., Refs. [4,17]), but are of course much more complicated and difficult to validate and implement, and especially to incorporate as retrofits into existing hydrodynamics codes.

By analogy with conventional descriptions of molecular and turbulent diffusion of materials, the required constitutive relations for the mass fluxes have usually been taken to be diffusional in character, so that $\mathbf{J}_{A}$ and $\mathbf{J}_{B}$ are proportional to concentration gradients. However, this analogy seems specious in the present context, since mixing due to interfacial instabilities is largely if not primarily inertial in character [15]. This inertial behavior is indeed an essential aspect of the dynamical ODE models for $h(t)$, and should be consis- 
tently retained in the constitutive relations for the mass fluxes as well. Our purpose here is to construct alternative constitutive relations which properly capture the dynamical/ inertial aspects of the mixing. To this end, we systematically exploit the fact that the constitutive relations for the mass fluxes determine, via the continuity equations, the concentration profiles through the mixing layer. We show that in sufficiently simple situations, this causal relationship can be analytically inverted to obtain the explicit form of the constitutive relations as a functional of the concentration profiles. This allows the former to be inferred from the latter, which are directly accessible via experiments or direct numerical simulations.

The simplifications upon which the present development is based are as follows:

(a) We restrict attention to planar interfaces between immiscible incompressible fluids with negligible surface tension. This restriction is common to much of the previous theoretical and experimental work in this area. Note that it implies the absence of molecular diffusion, so that mixing in the present context is entirely due to the mutual interpenetration of the two fluids produced by the instability.

(b) We further restrict attention to situations in which the mixing layer is characterized by a single integral length scale $h(t)$, which is presumed to be provided by a suitable mix or turbulence model and hence may be regarded as known for present purposes. This length scale is usually identified with the bubble penetration depth of the lighter fluid into the heavier one, but it may equally well be taken to be any other length scale of the same order of magnitude.

It should be noted that simplifications (a) and (b) are quite different in character, since (a) can be imposed experimentally, whereas the conditions, if any, under which (b) is valid are not at present known. We shall not attempt to address this issue, which is clearly related to the controversial and still unresolved question of the degree to which the length scale or wave number spectrum of the initial interfacial perturbation is "forgotten" as the instability evolves [18-20]. Resolution of this question will require further theoretical and experimental investigation. In the interim, however, we note that restriction (b) may be obeyed closely enough to provide a useful approximation even when it is not rigorously valid.

The first restriction (a) implies that $\rho_{A}$ and $\rho_{B}$ are completely determined by the ensemble averaged volume fraction $\alpha$ of the lighter fluid $A$, via the relations summarized in Sec. II. Moreover, $\alpha$ depends, by symmetry, only on the time $t$ and the spatial coordinate $x$ in the direction normal to the mixing layer. [If the system is spatially ergodic, so to speak, ensemble averages may be replaced by area averages over a plane of constant $x$; in either case $\alpha=\alpha(x, t)$.] The second restriction (b) further implies that $\alpha(x, t)$ depends on $x$ and $t$ only through the similarity variable $s=x / h(t)$; i.e., the volume fraction profile is self-similar in the variable $s$. This self-similarity and the resulting reduction in the number of independent variables is the crucial simplification that allows the relation between $\alpha$ and $\mathbf{J}_{A}$ to be inverted and solved for $\mathbf{J}_{A}$. The resulting constitutive relations for $\mathbf{J}_{A}$ and $\mathbf{J}_{B}=-\mathbf{J}_{A}$ can then be employed, as an extrapolation, in more complicated situations where the concentration profiles are not known a priori, such as problems lacking planar symmetry or involving compression. Such extrapolations of course represent approximations, which one would intuitively expect to be accurate in some neighborhood of the conditions defined by (a) and (b). However, the size and boundaries of that neighborhood can only be determined empirically by comparisons with further experiments and direct numerical simulations.

The paper is organized as follows. Section II summarizes the continuity equations and various basic relations between the partial mass densities and the corresponding volume fractions and mass fluxes of the two fluids, including an expression for the mass fluxes in terms of the relative velocity $\Delta \mathbf{u} \equiv \mathbf{u}_{A}-\mathbf{u}_{B}$. These relations are then specialized to the conditions defined by restriction (a) above, in which we obtain an evolution equation for $\alpha(x, t)$ in terms of $\Delta u(x, t)$. This equation is then transformed, by a change of variables, into a characteristic relationship between $\Delta u$ and the velocity of a surface of constant $\alpha$; i.e., $v_{\alpha} \equiv(\partial x / \partial t)_{\alpha}$. In Sec. III we invoke restriction (b), which implies that $\alpha$ is self-similar as discussed above. This in turn allows evaluation of $v_{\alpha}$, so that the characteristic equation involving $\Delta u$ can immediately be integrated to obtain an explicit expression for $\Delta u$ as a function of $(\alpha, t)$ and the self-similar volume fraction profile $\alpha(s)$. This then determines the mass fluxes $J_{A}$ and $J_{B}=-J_{A}$, which are simply proportional to $\Delta u$. In Sec. IV we show that the volumetric flow rates of the two fluids imply certain constraints on the form of $\alpha(s)$, which is an important technical detail. In Sec. $\mathrm{V}$ we show that even though $\Delta u$ is not inherently diffusional in character, it can nevertheless be transformed into an equivalent diffusional form which may be advantageous for numerical implementation. Section VI generalizes the main development of Secs. II-IV to situations in which the light and heavy regions of the mixing layer are characterized by two independent integral length scales $h_{1}(t)$ and $h_{2}(t)$ that depend differently on time. Section VII contains a few concluding remarks. A test calculation confirming the validity and practical utility of the formulation is presented in an Appendix.

\section{CONTINUITY EQUATIONS AND MASS FLUXES}

The mass densities $\rho_{A}$ and $\rho_{B}$ are partial densities; i.e., masses of fluids $A$ and $B$ per unit total volume of the mixture. Consequently, they each satisfy a continuity equation of the usual form

$$
\begin{gathered}
\frac{\partial \rho_{A}}{\partial t}+\nabla \cdot\left(\rho_{A} \mathbf{u}_{A}\right)=0, \\
\frac{\partial \rho_{B}}{\partial t}+\nabla \cdot\left(\rho_{B} \mathbf{u}_{B}\right)=0 .
\end{gathered}
$$

It is noteworthy that in this form, these equations apply equally well to both molecular and multiphase mixtures. It is the latter interpretation which is appropriate here, since molecular diffusion is absent in the present context. It is also noteworthy that in contrast to the other evolution equations, the local instantaneous and ensemble averaged continuity equations look formally identical because the averaged fluid 
velocities are mass weighted. Thus, for example, the mean velocity of fluid $A$ is not defined by $\left\langle\mathbf{u}_{A}\right\rangle$ (where $\langle Q\rangle$ denotes the ensemble average of the local instantaneous quantity $Q$ ), but rather by $\left\langle\rho_{A} \mathbf{u}_{A}\right\rangle /\left\langle\rho_{A}\right\rangle$. [Indeed, interpreting $\mathbf{u}_{A}$ in Eq. (4) as $\left\langle\mathbf{u}_{A}\right\rangle$ would not even be sensible, since the local instantaneous value of $\mathbf{u}_{A}$ is undefined at points outside fluid $A$.] In the present context, all equations and the quantities appearing therein are to be interpreted in an ensemble (or area) averaged sense, and this will henceforth be understood.

In situations where the mass-weighted mean velocity $\mathbf{u}$ is determined by a single momentum equation for the mixture as a whole, it is appropriate and customary to rewrite Eqs. (4) and (5) in the equivalent form

$$
\begin{aligned}
& \frac{\partial \rho_{A}}{\partial t}+\boldsymbol{\nabla} \cdot\left(\rho_{A} \mathbf{u}\right)=-\boldsymbol{\nabla} \cdot \mathbf{J}_{A}, \\
& \frac{\partial \rho_{B}}{\partial t}+\boldsymbol{\nabla} \cdot\left(\rho_{B} \mathbf{u}\right)=-\boldsymbol{\nabla} \cdot \mathbf{J}_{B},
\end{aligned}
$$

where use has been made of Eqs. (2) and (3). Equations (6) and (7) show clearly that constitutive relations for $\mathbf{J}_{A}$ and $\mathbf{J}_{B}$ are needed to close the system. These fluxes are simply proportional to the relative velocity $\Delta \mathbf{u} \equiv \mathbf{u}_{A}-\mathbf{u}_{B}$ of the lighter fluid relative to the heavier one, as can be seen by combining Eqs. (1)-(3) to obtain

$$
\mathbf{J}_{A}=-\mathbf{J}_{B}=\left(\frac{\rho_{A} \rho_{B}}{\rho}\right) \Delta \mathbf{u} .
$$

Knowledge of $\Delta \mathbf{u}$ is therefore sufficient to obtain the mass fluxes, so we may henceforth restrict our attention to determining $\Delta \mathbf{u}$.

Since we are regarding the mixing layer as a two-phase mixture of fluids $A$ and $B$, the fluid volume fractions $\alpha_{A}$ $\equiv \alpha$ and $\alpha_{B}=1-\alpha_{A}=1-\alpha$ are well defined, and are again to be interpreted as ensemble averages. The mean mass densities of the pure fluids $A$ and $B$ (i.e., the mass of each fluid per unit volume occupied by that fluid) are then given by

$$
\rho_{A}^{0}=\frac{\rho_{A}}{\alpha}, \quad \rho_{B}^{0}=\frac{\rho_{B}}{1-\alpha}
$$

and, conversely,

$$
\rho_{A}=\alpha \rho_{A}^{0}, \quad \rho_{B}=(1-\alpha) \rho_{B}^{0} .
$$

Combining Eqs. (4), (5), and (10), we obtain

$$
\begin{gathered}
\frac{\partial\left(\alpha_{A} \rho_{A}^{0}\right)}{\partial t}+\boldsymbol{\nabla} \cdot\left(\alpha_{A} \rho_{A}^{0} \mathbf{u}_{A}\right)=0, \\
\frac{\partial\left(\alpha_{B} \rho_{B}^{0}\right)}{\partial t}+\boldsymbol{\nabla} \cdot\left(\alpha_{B} \rho_{B}^{0} \mathbf{u}_{B}\right)=0
\end{gathered}
$$

which are simply the standard two-phase continuity equations in their conventional form [21,22].

We now proceed to specialize the above general relations to the conditions defined by simplification (a). The restriction to incompressible fluids implies that the pure fluid densities $\rho_{A}^{0}$ and $\rho_{B}^{0}$ become known given constants which can be taken outside of space and time derivatives. It then follows from Eq. (10) that knowledge of $\alpha$ as a function of position and time uniquely determines the partial densities $\rho_{A}$ and $\rho_{B}$ and the total density $\rho$. The restriction to planar geometry further reduces the problem to one space dimension, so that vectors reduce to scalars and the divergence reduces to $\partial / \partial x$. Equations (11) and (12) then become

$$
\begin{aligned}
& \frac{\partial \alpha_{A}}{\partial t}+\frac{\partial}{\partial x}\left(\alpha_{A} u_{A}\right)=0, \\
& \frac{\partial \alpha_{B}}{\partial t}+\frac{\partial}{\partial x}\left(\alpha_{B} u_{B}\right)=0 .
\end{aligned}
$$

Adding Eqs. (13) and (14), we obtain

$$
\frac{\partial u_{v}}{\partial x}=0
$$

where $u_{v} \equiv \alpha_{A} u_{A}+\alpha_{B} u_{B}$ is the volume-weighted mean velocity of the mixture [23], which differs from the ordinary massweighted mean velocity $u$. Equation (15) shows that $u_{v}$ is independent of $x$ and is therefore a function of time alone; i.e., $u_{v}=u_{v}(t)$.

Now $u_{A}=u_{v}+\left(u_{A}-u_{v}\right)$, and $u_{A}-u_{v}=u_{A}-\alpha_{A} u_{A}-\alpha_{B} u_{B}$ $=\alpha_{B} \Delta u$, so Eq. (13) may be rewritten as

$$
\frac{\partial \alpha}{\partial t}+u_{v} \frac{\partial \alpha}{\partial x}=-\frac{\partial}{\partial x}[\alpha(1-\alpha) \Delta u]
$$

which may be regarded as the fundamental time evolution equation for $\alpha(x, t)$ in an incompressible planar mixing layer. We shall suppose that the lighter fluid $A$ initially occupies the region $-\infty<x<0$, while the heavier fluid $B$ occupies the region $0<x<\infty$, so that $\alpha(x, t)$ approaches unity as $x \rightarrow-\infty$ and zero as $x \rightarrow \infty$. This in turn implies that $u_{v}=u_{A}(-\infty, t)$ $=u_{B}(\infty, t)$, so that $u_{v}(t)$ is also the common velocity of the pure fluids far from the mixing layer. Since the fluids are incompressible, this is also the velocity with which the mixing layer itself translates in the $x$ direction. It is convenient to choose a reference frame or coordinate system in which the fluids are stationary at infinity, so that $u_{v}=0$ and the mixing layer grows in width but does not translate while doing so. Equation (16) then becomes

$$
\frac{\partial \alpha}{\partial t}=-\frac{\partial}{\partial x}[\alpha(1-\alpha) \Delta u] .
$$

Now one would intuitively expect that in fully developed mixing layers, $\alpha(x, t)$ will be a monotonically decreasing function of $x$ at constant $t$, and this behavior has indeed been observed in a wide variety of experimental and computational studies $[2,4,14,18,19,24-26]$. Slight deviations from monotonicity have occasionally been reported in numerical simulations (e.g., Refs. [2,24]), but seem likely to have been artifacts of inadequate statistics, residual memory of initial conditions at early times, and/or discretization errors. In any case, it is clear that volume fraction profiles in planar mixing layers are normally monotonic, and this behavior will be assumed in what follows. 
The relative velocity $\Delta u$ is fundamentally a function of $(x, t)$, but the assumed monotonicity of $\alpha(x, t)$ implies that $x$ is conversely a single-valued monotonically decreasing function of $\alpha$ at constant $t$, so that we may equally well regard $\Delta u$ as a function of $(\alpha, t)$ instead. Thus we write $\Delta u=\Delta u(\alpha, t)$, and this functional dependence will henceforth be understood. Using the chain rule to convert from $\partial / \partial x$ to $\partial / \partial \alpha$, we may then rewrite Eq. (17) in the form

$$
\frac{\partial}{\partial \alpha}[\alpha(1-\alpha) \Delta u]=-\frac{\partial \alpha / \partial t}{\partial \alpha / \partial x}=\left(\frac{\partial x}{\partial t}\right)_{\alpha} \equiv v_{\alpha},
$$

where use has been made of a well-known identity involving partial derivatives. Equation (18) is essentially a special case of a basic relation in the characteristic analysis of a firstorder nonlinear hyperbolic wave equation of conservation form [27].

\section{SCALING AND SELF-SIMILARITY}

We now proceed to explore the consequences of simplification (b), which implies that the only length scales contained in $\alpha(x, t)$, or upon which $\alpha(x, t)$ can in any way depend, are proportional to $h(t)$. This in turn implies that any velocity that can be extracted from $\alpha(x, t)$ must be proportional to $\dot{h} \equiv d h / d t$. This applies, in particular, to $v_{\alpha}$, so that

$$
v_{\alpha}=\left(\frac{\partial x}{\partial t}\right)_{\alpha}=F(\alpha) \dot{h}(t),
$$

where $F(\alpha)$ is an as yet undetermined function of $\alpha$ alone. Integrating from $t=0$ to $t$ at constant $\alpha$, we obtain

$$
x(\alpha, t)=x(\alpha, 0)+F(\alpha)[h(t)-h(0)] .
$$

But at $t=0$, the mixing layer is a discontinuity with zero thickness, so that $h(0)=0$ and $x(\alpha, 0)=0$ for any value of $\alpha$ between zero and unity. Equation (20) then simply reduces to $x=F(\alpha) h(t)$, or $F(\alpha)=x / h(t) \equiv s$, which implies that $\alpha$ is a function of $s$ alone. Thus $\alpha$ depends on $(x, t)$ only through $s$, and consequently exhibits self-similar scaling behavior in the similarity variable $s$. We may therefore write $\alpha=\alpha(s)$, and conversely $s=s(\alpha)$, where the function $s(\alpha)$ is the inverse of the function $\alpha(s)$, and is now seen to be identical to the previously undetermined function $F(\alpha)$. When $\alpha(s)$ is known in tabular form, $s(\alpha)$ is easily obtained simply by interchanging the two columns in the table.

Since $F(\alpha)=s(\alpha)$, Eq. (19) now becomes $v_{\alpha}=\dot{h}(t) s(\alpha)$, which combines with Eq. (18) to yield

$$
\frac{\partial}{\partial \alpha}[\alpha(1-\alpha) \Delta u]=\dot{h} s(\alpha) .
$$

Equation (21) can immediately be integrated, with the result

$$
\Delta u(\alpha, t)=\frac{\dot{h}(t)}{\alpha(1-\alpha)} \int_{0}^{\alpha} s\left(\alpha^{\prime}\right) d \alpha^{\prime}
$$

which explicitly determines the form of the relative velocity required to produce or reproduce a given self-similar volume fraction profile $\alpha(s)$. Equation (22) is our main result, which immediately determines the desired constitutive relations for the mass fluxes via Eq. (8).

The integral in Eq. (22) obviously vanishes as $\alpha \rightarrow 0$, and we shall show in the next section that it also vanishes as $\alpha$ $\rightarrow 1$. However, when the profile $\alpha(s)$ is not of finite extent [i.e., when $\alpha(1-\alpha)$ is nonzero for all finite values of $s$ ], application of L'Hôpital's rule to Eq. (22) shows that $\Delta u(\alpha, t)$ will correspondingly diverge as $\alpha \rightarrow 0$ or 1 . This behavior is of course unphysical, but is merely a harmless artifact of the fact that profiles of this type, while mathematically convenient, imply an infinite signal speed and are hence themselves unphysical. The divergence of $\Delta u$ as $\alpha$ $\rightarrow 0$ or 1 in such cases simply reflects the fact that an arbitrarily large relative velocity is required to produce a finite disturbance arbitrarily far from the initial interface at a finite time.

\section{VOLUMETRIC CONSTRAINTS}

We have loosely referred to the profile $\alpha(s)$ as "arbitrary," but strictly speaking this is not quite true: it must be properly centered volumetrically, so that equal volumes of the two fluids lie on each side of the point $s=0$. This constraint is a consequence of the fact that we are in a coordinate system where $u_{v}=0$, which implies that there is no net flow of volume across any surface of constant $x$, in particular the surface $x=0$ at which $s=0$ for all time. The volumetric flow rates of fluids $A$ and $B$ across this surface must therefore always be equal and opposite, and since this surface separates the two fluids at $t=0$, it is clear that at any later time, the total volume of fluid $B$ in the region $x<0$ must be the same as the total volume of fluid $A$ in the region $x>0$, i.e.,

$$
\int_{-\infty}^{0}[1-\alpha(x, t)] d x=\int_{0}^{\infty} \alpha(x, t) d x .
$$

This relation is easily confirmed mathematically by integrating Eqs. (13) and (14) over the regions $0<x<\infty$ and $-\infty$ $<x<0$, respectively, subtracting the results, and integrating with respect to time. Integrating by parts, we obtain the simpler equivalent relation

$$
\int_{-\infty}^{\infty} x \frac{\partial \alpha}{\partial x} d x=0
$$

Transforming the integration variables from $x$ to $s$ at constant $t$, we obtain the equivalent constraints on the profile $\alpha(s)$,

$$
\int_{-\infty}^{0}[1-\alpha(s)] d s=\int_{0}^{\infty} \alpha(s) d s
$$

and

$$
\int_{-\infty}^{\infty} s \frac{d \alpha}{d s} d s=0
$$

Transforming the integration variable in Eq. (26) from $s$ to $\alpha$ then yields the equivalent constraint on the inverse profile $s(\alpha)$, 


$$
\int_{0}^{1} s(\alpha) d \alpha=0 .
$$

As a simple example, we consider the case in which the profile $\alpha(s)$ is linear in $s$, which is the simplest qualitatively reasonable possibility. This was the special case considered in Ref. [15], where $\Delta u$ was taken to be proportional to $\dot{h}$ but independent of $\alpha$ or $x$. Let us confirm that Eq. (22) in fact yields a $\Delta u$ of that form for this case. We therefore suppose that $\alpha(s)$ varies linearly from a value of unity at $s=a<0$ to a value of zero at $s=b>0$. The inverse profile $s(\alpha)$ then varies linearly from $b$ at $\alpha=0$ to $a$ at $\alpha=1$, and therefore has the form $s(\alpha)=b+(a-b) \alpha$. The volumetric constraint of Eq. (27) then implies that $a+b=0$, so that $a=-b$. The inverse profile $s(\alpha)$ then becomes $s(\alpha)=b(1-2 \alpha)$, which combines with Eq. (22) to yield $\Delta u(\alpha, t)=b \dot{h}(t)$. A relative velocity proportional to $\dot{h}$ but independent of $\alpha$ or $x$ therefore corresponds to a linear volume fraction profile, and vice versa, just as was found in Ref. [15].

In most cases, one will wish to infer $\alpha(s)$ from some given "raw" volume fraction profile $\alpha_{0}\left(x^{\prime}, t_{0}\right)$ at a fixed time $t_{0}$, with a corresponding length scale $h=h\left(t_{0}\right) \equiv h_{0}$. The independent variable in the raw profile has been denoted by $x^{\prime}$ rather than $x$ to allow for the possibility that $\alpha_{0}$ may have been determined in a coordinate system with a different origin from that assumed in the present development. If so, the two coordinate systems are simply related at time $t_{0}$ by a shift transformation of the form $x^{\prime}=x+x_{0}$, where $x_{0}$ may easily be determined by requiring the raw profile as a function of $x$, namely, $\alpha_{0}\left(x+x_{0}, t_{0}\right)$, to satisfy the volumetric constraint conditions derived above, in particular Eq. (24). Once $x_{0}$ has been determined in this way, $\alpha(s)$ may simply be identified with $\alpha_{0}\left(h_{0} s+x_{0}, t_{0}\right)$. However, it is more convenient to derive the equivalent relation for $s(\alpha)$, since this is the quantity appearing in the constitutive relations. To this end, we define $s^{\prime} \equiv x^{\prime} / h(t)=x^{\prime} / h_{0}$ at $t=t_{0}$, a function $\alpha\left(s^{\prime}\right)$ $\equiv \alpha_{0}\left(h_{0} s^{\prime}, t_{0}\right)$, and the corresponding inverse function $s^{\prime}(\alpha)$. Thus the functions $\alpha\left(s^{\prime}\right)$ and $s^{\prime}(\alpha)$ are simply the self-similar profile and inverse profile that one would naively infer from the raw profile by ignoring the volumetric constraints, and they will not in general satisfy those constraints. Now at time $t_{0}$,

$$
s^{\prime}(\alpha)=\frac{x^{\prime}}{h_{0}}=\frac{x+x_{0}}{h_{0}}=s(\alpha)+s_{0},
$$

where $s_{0}=x_{0} / h_{0}$. Equation (28) combines with Eq. (27) to yield

$$
s_{0}=\int_{0}^{1} s^{\prime}(\alpha) d \alpha,
$$

whereupon Eq. (28) becomes

$$
s(\alpha)=s^{\prime}(\alpha)-\int_{0}^{1} s^{\prime}\left(\alpha^{\prime}\right) d \alpha^{\prime} .
$$

Equation (30) determines the corrected and volumetrically consistent inverse profile $s(\alpha)$ in terms of the corresponding volumetrically inconsistent inverse profile $s^{\prime}(\alpha)$. The resulting $s(\alpha)$ manifestly satisfies the volumetric constraint condition of Eq. (27).

\section{EQUIVALENT DIFFUSIONAL FORMULATION}

As was anticipated in the Introduction, Eq. (22) for $\Delta u$ is, or at least superficially appears to be, manifestly nondiffusional in nature, since it is algebraic in form rather than being proportional to concentration gradients. However, we now proceed to show that Eq. (22) for $\Delta u$ can nevertheless be transformed into an alternative equivalent expression which is diffusional in form, in spite of the fact that the underlying physical process determining $h(t)$ is not inherently or fundamentally diffusional in character. As would be expected on dimensional grounds, the diffusion coefficient in this expression is found to be of the form $D=h \dot{h} C(\alpha)$, and we shall derive an explicit analytical expression for the dimensionless function $C(\alpha)$ as a functional of $s(\alpha)$. The simple case in which $C$ is a constant independent of $\alpha$ will be shown to correspond to a volume fraction profile $\alpha(s)$ of error function form, and vice versa.

Now if the mass fluxes $\mathbf{J}_{A}$ and $\mathbf{J}_{B}$ were indeed diffusional in character, they would have the Fick's Law form [28]

$$
\mathbf{J}_{A}=-\mathbf{J}_{B}=-\rho D \nabla\left(\frac{\rho_{A}}{\rho}\right) .
$$

When the two fluids $A$ and $B$ are incompressible, Eq. (31) readily reduces to

$$
\mathbf{J}_{A}=-\mathbf{J}_{B}=-\left(\frac{\rho_{A}^{0} \rho_{B}^{0}}{\rho}\right) D \nabla \alpha,
$$

where use has been made of Eq. (10). Comparison with Eq. (8) shows that diffusional mass fluxes of this form correspond to a relative velocity of the form

$$
\alpha(1-\alpha) \Delta \mathbf{u}=-D \nabla \alpha
$$

which in the present one-dimensional context reduces to

$$
\alpha(1-\alpha) \Delta u=-D \frac{\partial \alpha}{\partial x}
$$

Since $\alpha(x, t)=\alpha(s)$, Eq. (34) further reduces to

$$
\alpha(1-\alpha) \Delta u=-\frac{D}{h} \frac{d \alpha}{d s} .
$$

Comparison of Eq. (35) with the previous nondiffusional Eq. (22) for $\Delta u$ then shows that the two can indeed be made consistent simply by requiring that

$$
-\frac{D}{h} \frac{d \alpha}{d s}=\dot{h} \int_{0}^{\alpha} s\left(\alpha^{\prime}\right) d \alpha^{\prime} .
$$

Solving for the diffusion coefficient $D$, we find

$$
D=h \dot{h} C(\alpha),
$$

where 


$$
C(\alpha)=-\frac{d s(\alpha)}{d \alpha} \int_{0}^{\alpha} s\left(\alpha^{\prime}\right) d \alpha^{\prime} .
$$

Equations (37) and (38) provide an explicit expression for the diffusion coefficient $D$ required to produce or reproduce any given self-similar volume fraction profile $\alpha(s)$. Thus, contrary to superficial appearances, the diffusional form of $\Delta u$ given in Eq. (34) is in fact consistent with and equivalent to the algebraic expression for $\Delta u$ given in Eq. (22), provided that the diffusion coefficient is chosen appropriately.

Of course, this result is potentially useful only in situations where the resulting $D$ is positive, since otherwise the diffusional formulation would be catastrophically unstable to small perturbations and would represent an ill-posed initial value problem. Now $h>0$ by definition, and it is straightforward to show that $C>0$ by virtue of Eq. (27) and the monotonicity of $\alpha(s)$. The sign of $D$ is therefore the same as that of $\dot{h}$, so that $D$ will always be positive when $\dot{h}>0$; i.e., when the mixing layer is growing or expanding with time. Conversely, however, if the mixing layer is shrinking or contracting (i.e., the two fluids are separating or demixing) then $\dot{h}$ $<0$, which implies $D<0$. In this case Eqs. (31), (37), and (38) should not be used, and the original Eqs. (8) and (22) should be used instead.

It is of interest to determine the volume fraction profile for which $C$ is a constant independent of $\alpha$. To this end, we rewrite Eq. (38) for constant $C$ in the form

$$
C \frac{d \alpha}{d s}=-\int_{0}^{\alpha} s\left(\alpha^{\prime}\right) d \alpha^{\prime} .
$$

Differentiating Eq. (39) with respect to $s$, we obtain

$$
C \frac{d^{2} \alpha}{d s^{2}}=-s \frac{d \alpha}{d s}
$$

which is easily integrated to yield

$$
\frac{d \alpha}{d s}=A \exp \left(-\frac{s^{2}}{2 C}\right),
$$

where $A$ is the value of $d \alpha / d s$ at $s=0$. Integrating Eq. (41) from $s=-\infty$ (where $\alpha=1)$ to $s$, we obtain

$$
\alpha(s)=1+A \sqrt{\frac{\pi C}{2}}\left[1+\operatorname{erf}\left(\frac{s}{\sqrt{2 C}}\right)\right],
$$

where $\operatorname{erf}(z)$ is the standard error function. The value of $A$ is determined by the requirement that $\alpha(s) \rightarrow 0$ as $s \rightarrow \infty$. We thereby obtain $A=-1 / \sqrt{2 \pi C}$, and Eq. (42) then becomes

$$
\alpha(s)=\frac{1}{2}\left[1-\operatorname{erf}\left(\frac{s}{\sqrt{2 C}}\right)\right]
$$

which not surprisingly has the well-known error function form that typically occurs in self-similar diffusion problems [28]. Thus a volume fraction profile of error function form corresponds to a constant value of $C$ and hence a diffusion coefficient $D$ independent of $\alpha$, and vice versa.

We reemphasize that the above development does not in any sense imply that the underlying physical process produc- ing $\alpha(s)$ is inherently diffusional in character. We have simply shown that an arbitrary monotonic $\alpha(s)$, whatever its physical origin, can be reproduced (or mimicked, as it were) by a $J_{A}$ or $\Delta u$ of diffusional form, provided the diffusion coefficient $D$ is appropriately chosen as a function of time and the local value of $\alpha$. This lack of an intrinsic physical interpretation is further emphasized by the observation that we could equally well construct, by an entirely similar argument, an infinity of other alternative equivalent expressions for $J_{A}$ in terms of various arbitrary functions of the form $d^{n} G(\alpha) / d x^{n}$ having no physical significance. Which of these various analytically equivalent alternatives one chooses to work with is entirely a matter of mathematical convenience. In this regard, the diffusional formulation presented above seems likely to be especially convenient and well suited for numerical or computational purposes, where it has the following apparent advantages: (a) it may tend to be more stable and well behaved numerically, since diffusion is a smoothing process, (b) it should be easier to retrofit into existing hydrodynamics codes which already transport materials diffusionally, and (c) it is more straightforward to implement multidimensionally, where $\partial \alpha / \partial x \rightarrow \nabla \alpha$. This automatically produces a vectorial $\mathbf{J}_{A}$ or $\Delta \mathbf{u}$ pointing in the direction normal to the mixing layer, thereby eliminating the previous need to determine this direction separately [15]. Moreover, the error function volume fraction profile implied by the simple approximation of constant $C$ is qualitatively more realistic than the linear profile implied by the approximation that $\Delta u$ is independent of $\alpha$. This in turn suggests that $D$ is in general likely to be a weaker function of $\alpha$ than $\Delta u$ is.

Zhou et al. [29] recently described a different procedure for defining a variable diffusion coefficient proportional to $h \dot{h}$ within the mixing layer. Their procedure contains an adjustable parameter which allows the user to vary the volume fraction profile to some extent, but it was not designed to accommodate or reproduce an arbitrary specified profile.

\section{PIECEWISE SIMILARITY}

The preceding development presumes that the entire volume fraction profile exhibits self-similar scaling behavior based on a single integral scale length $h(t)$. However, when the density ratio $\rho_{B}^{0} / \rho_{A}^{0}$ becomes sufficiently large and the acceleration history varies sufficiently rapidly in time, there is considerable evidence that the spike and bubble penetration depths no longer exhibit the same time dependence [12-14]. The light and heavy sides of the mixing layer are then characterized by independent integral length scales $h_{1}(t)$ and $h_{2}(t)$. It is convenient and customary to identify these length scales with the corresponding penetration depths, and this will henceforth be understood. Some mix models accordingly determine $h_{1}(t)$ and $h_{2}(t)$ by means of separate evolution equations, which are generally similar in form but with different coefficients (e.g., Refs. $[13,14]$ ). In this situation, global self-similarity of the type discussed above is clearly no longer possible. However, the preceding development can be generalized to accommodate this situation by constructing piecewise self-similar volume fraction profiles 
based on the assumption that $\alpha$ exhibits different self-similar scaling behavior in the light and heavy regions of the mixing layer. As will be seen, however, these regions can no longer simply be defined by $x<0$ and $x>0$, but must rather be defined by $x<X(t)$ (region 1) and $x>X(t)$ (region 2), where $X(t)$ is implicitly determined by the volumetric constraint condition of Eq. (23). This further implies that for present purposes, $h_{1}(t)$ and $h_{2}(t)$ should not be defined in terms of penetration depths relative to $x=0$, which is the standard convention, but rather in terms of penetration depths relative to $x=X(t)$. Fortunately, the conversion between these two definitions is straightforward. We thereby obtain a generalized expression for $\Delta u$ suitable for use in conjunction with mix models that determine $h_{1}(t)$ and $h_{2}(t)$ by means of separate evolution equations.

We accordingly introduce a generalized similarity variable $s$ defined by $s=[x-X(t)] / h_{1}(t)$ for $x<X(t)$ and $s=[x$ $-X(t)] / h_{2}(t)$ for $x>X(t)$, and we again assume that $\alpha(x, t)$ depends on $x$ and $t$ only through $s$. Thus we still have $\alpha$ $=\alpha(s)$ and $s=s(\alpha)$ as before, but the transformation between the variables $x$ and $s$ becomes somewhat more complicated. Performing this transformation in the volumetric constraint condition of Eq. (23), we obtain, after a little algebra,

$$
X(t)=h_{1}(t) I_{1}-h_{2}(t) I_{2},
$$

where

$$
\begin{gathered}
I_{1}=\int_{-\infty}^{0}[1-\alpha(s)] d s=-\int_{\alpha_{0}}^{1} s(\alpha) d \alpha, \\
I_{2}=\int_{0}^{\infty} \alpha(s) d s=\int_{0}^{\alpha_{0}} s(\alpha) d \alpha,
\end{gathered}
$$

and $\alpha_{0} \equiv \alpha(0)$ is the time-independent value of $\alpha$ at $s=0$; i.e., at $x=X(t)$. Equation (44) determines $X(t)$ in terms of the profile $\alpha(s)$. Notice that if we had assumed $X=0$ at the outset, it would have been impossible to satisfy Eq. (44) unless $h_{1}(t) / h_{2}(t)=$ const; i.e., unless the two scale lengths had the same time dependence. If the scale lengths $h_{i}(t)$ are defined as penetration depths relative to $x=X(t)$, then the corresponding penetration depths relative to $x=0$ are simply

$$
h_{1}^{*}(t)=h_{1}(t)-X(t), \quad h_{2}^{*}(t)=h_{2}(t)+X(t) .
$$

It is the quantities $h_{i}^{*}(t)$ that should be identified with the penetration depths in conventional ODE mix models. Conversely,

$$
h_{1}(t)=h_{1}^{*}(t)+X(t), \quad h_{2}(t)=h_{2}^{*}(t)-X(t)
$$

which combines with Eq. (44) to yield

$$
X(t)=\frac{h_{1}^{*}(t) I_{1}-h_{2}^{*}(t) I_{2}}{1-I_{1}-I_{2}} .
$$

Equation (49) expresses $X(t)$ in terms of the $h_{i}^{*}(t)$, whereupon Eq. (48) provides an explicit expression for the $h_{i}(t)$ in terms of the $h_{i}^{*}(t)$.

We are now in a position to carry through the development of Sec. III in a piecewise fashion, allowing for the different relations between $x$ and $s$ in the two regions. Equation (21) is now replaced by

$$
\frac{\partial}{\partial \alpha}[\alpha(1-\alpha) \Delta u]=\left(\frac{\partial x}{\partial t}\right)_{s}=\dot{h}_{i} s(\alpha)+\dot{X}
$$

in which $i=1$ for $\alpha>\alpha_{0}$ and $i=2$ for $\alpha<\alpha_{0}$, or conversely $\alpha>\alpha_{0}$ for $i=1$ and $\alpha<\alpha_{0}$ for $i=2$. In either case, Eq. (50) may be integrated from $\alpha_{0}$ to $\alpha$, with the result

$$
\alpha(1-\alpha) \Delta u=\alpha_{0}\left(1-\alpha_{0}\right) \Delta u_{0}+\dot{h}_{i} \int_{\alpha_{0}}^{\alpha} s\left(\alpha^{\prime}\right) d \alpha^{\prime}+\dot{X}\left(\alpha-\alpha_{0}\right),
$$

where $\Delta u_{0}=\Delta u\left(\alpha_{0}, t\right)$. Evaluating Eq. (51) at $\alpha=0$, where $i$ $=2$, we obtain

$$
\alpha_{0}\left(1-\alpha_{0}\right) \Delta u_{0}=\dot{h}_{2} I_{2}+\dot{X} \alpha_{0},
$$

where use has been made of Eq. (46). Combining Eqs. (44), (51), and (52), we finally obtain

$$
\begin{aligned}
\Delta u(\alpha, t)= & \frac{1}{\alpha(1-\alpha)}\left[\dot{h}_{i}(t) \int_{\alpha_{0}}^{\alpha} s\left(\alpha^{\prime}\right) d \alpha^{\prime}+\alpha \dot{h}_{1}(t) I_{1}\right. \\
& \left.+(1-\alpha) \dot{h}_{2}(t) I_{2}\right]
\end{aligned}
$$

where again $i=1$ for $\alpha>\alpha_{0}$ and $i=2$ for $\alpha<\alpha_{0}$. Equation (53) provides an explicit expression for the $\Delta u(\alpha, t)$ required to produce or reproduce any specified piecewise self-similar volume fraction profile $\alpha(s)$.

Finally, we remark that the present case of piecewise similarity can also be readily transformed into an alternative equivalent diffusional formulation of the type developed in the preceding section. This transformation is straightforward, and will therefore be omitted in the interests of brevity.

\section{CONCLUDING REMARKS}

We have shown how to construct explicit analytical constitutive relations for the mass fluxes $\mathbf{J}_{A}$ and $\mathbf{J}_{B}$ in planar incompressible mixing layers in such a way as to reproduce any known or given monotonic self-similar volume fraction profile $\alpha(s)$. The latter profiles are directly accessible via experiments or direct numerical simulations, whereas the constitutive relations themselves are not. The present development provides a means by which the latter may be inferred from the former. Once the constitutive relations have been determined in this way, they may be employed as an extrapolation in other situations where the volume fraction profiles are not known a priori, such as problems lacking spatial symmetry or perhaps even problems involving compression. Such extrapolations, of course, represent approximations whose accuracy can only be determined by comparision with experiments or direct numerical simulations. These constitutive relations will no doubt be more accurate in some problems than others, but at least they are guaranteed by construction to produce the correct volume fraction profiles in the special case of planar incompressible mixing layers. 
The present results for $\mathbf{J}_{A}$ and $\mathbf{J}_{B}$ must be used in conjunction with a suitable mix model to determine the length scale $h(t)$. The formalism itself is compatible with any such model, but the accuracy of the results will of course be limited by that of the model from which $h(t)$ is obtained.

The form of these constitutive relations is well suited for numerical implementation in hydrodynamics codes, thereby allowing the simulation of problems involving material mixing due to unresolved (subgrid scale) perturbations on unstable fluid interfaces [15]. However, the present development has been based upon the essential assumption that $h(t)$ is a known function of time alone, whereas implementation of mix and turbulence models in hydrodynamics codes will ordinarily produce length scales that depend on position as well as time $[15,16]$. This inconsistency is conceptually unsatisfactory, but unfortunately it cannot be entirely eliminated in descriptions of this type; it must simply be tolerated as an approximation, which seems intuitively reasonable as long as $h$ varies only slightly over distances of order $h$ itself. Such inconsistencies are not peculiar to the present context, but tend to arise whenever one attempts to interpret global or integral quantities such as $h$ as local variables. In particular, similar inconsistencies are also implicit in much of conventional two-phase flow and turbulence modeling [30], where they have yet to receive the attention they deserve.

\section{ACKNOWLEDGMENTS}

I am grateful to C. H. Chang for helpful discussions. A portion of this work was performed under the auspices of the U. S. Department of Energy by the University of California Lawrence Livermore National Laboratory under Contract No. W-7405-Eng-48.

\section{APPENDIX: TEST CALCULATION}

A test calculation was performed to verify that a straightforward numerical implementation of the constitutive relations derived herein will indeed accurately reproduce a given experimentally determined volume fraction profile. For this purpose, we used the nondiffusional form of Eq. (22) to further emphasize the fact that the mixing process is not inherently diffusional in nature.

The particular experimental volume fraction profile chosen for this calculation was that reported in Fig. 10(b) of Ref. [14]. That figure displays the volume fraction $1-\alpha$ of the heavier fluid as a function of the scaled distance $z / 2 A Z$ in a Rayleigh-Taylor experiment with Atwood number $A=0.32$ and acceleration $g \approx 70 g_{0}$, where $g_{0}=980 \mathrm{~cm} / \mathrm{s}^{2}, z$ is the normal distance coordinate denoted here by $x$, and $Z \approx 0.5 g t^{2}$ is the displacement of the interface at time $t$. This profile was manually digitized at a set of 33 discrete data points equally spaced in the independent variable $z / 2 A Z$. (The digitization was conveniently performed by scaling the cursor pixel coordinates displayed when viewing the figure with ghostview.) The resulting table of $1-\alpha$ vs. $z / 2 A Z$ was then converted into a corresponding table of $\alpha$ vs. $s=x / h$ by identifying $h$ with the bubble penetration depth $\alpha_{2} A g t^{2}$, so that $\alpha_{2} s$ $=z / 2 A Z$,

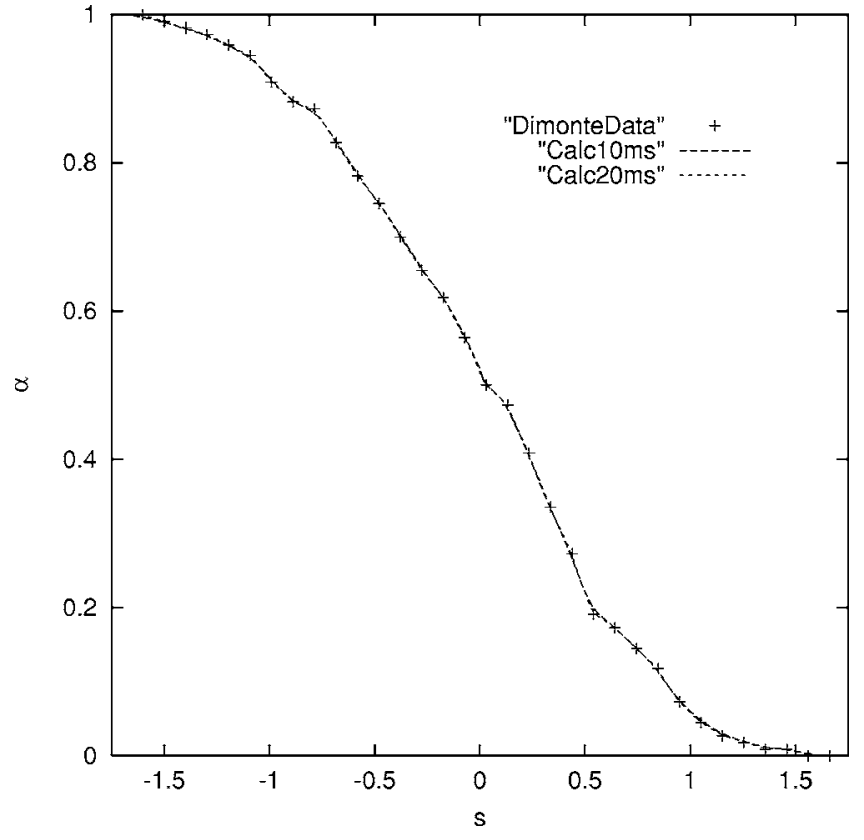

FIG. 1. Comparison of experimental volume fraction profile (+ points) and calculated profiles obtained by numerically solving Eq. (17) with $\Delta u$ given by Eq. (22) (dashed lines).

where $\alpha_{2}=0.049$ [14]. The discretization introduced slight errors into the volumetric constraint conditions of Sec. IV, so these errors were corrected by slightly shifting the $s$ values in the table as prescribed by Eq. (30). The resulting experimentally determined $\alpha(s)$ profile is shown by the "+" data points in Fig. 1. (The small irregularities in the profile are present in the original figure [14].) The values of the integral $I(\alpha)$ $\equiv \int_{0}^{\alpha} s\left(\alpha^{\prime}\right) d \alpha^{\prime}$ at the same discrete values of $s$ were then computed and tabulated by numerical integration using the trapezoidal rule.

We then proceeded to generate corresponding calculated volume fraction profiles by numerically solving Eq. (17), with $\Delta u$ given by Eq. (22), using the MacCormack two-step predictor-corrector scheme [31,32]. This calculation of course required the evaluation of $I(\alpha)$ at values of $\alpha$ intermediate between those in the table. These intermediate values of $I$ were obtained by quadratic interpolation between the tabulated values, in accordance with the piecewise linear approximation to $s(\alpha)$ upon which the trapezoidal rule is based. The calculational region was $-L / 2 \leqslant x \leqslant L / 2$, where $L=1.5 \mathrm{~cm}$, and was subdivided by 1001 equally spaced mesh points $x_{i}=-L / 2+(i-1) \Delta x$, where $\Delta x=L / 1000$. The calculation used a variable time step $\Delta t=0.1 \Delta x / v_{\max }$, where $v_{\max }$ $=\dot{h}(t)|s(1)|$ is the largest value of $\left|v_{\alpha}\right|$ in the computing mesh.

In this problem it is unnecessary to use an auxiliary mix or turbulence model to obtain $h(t)$, since $h(t)=\alpha_{2} A g t^{2}$, so that $\dot{h}=2 \alpha_{2} A g t=2 h / t$. Because of the spatial discretization, the initial volume fraction profile has a finite width of order $\Delta x$, so the calculation was started at the time $t=t_{0}$ at which $h=\Delta x$; i.e., $t_{0}=\sqrt{\Delta x /\left(\alpha_{2} A g\right)}$. The volume fraction profile at this time was taken to be $\alpha\left(x, t_{0}\right)=[1-\tanh (x / \Delta x)] / 2$. The calculation was run out to a final time of $t=20 \mathrm{~ms}$. The re- 
sulting profiles of $\alpha(s)$ at $t=10 \mathrm{~ms}$ and $t=20 \mathrm{~ms}$ are shown as the dashed curves in Fig. 1. Since $h$ is quadratic in $t$, the $\alpha(x, t)$ profile is four times wider at the latter time than the former one, but these profiles collapse onto a single curve when plotted vs. the similarity variable $s=x / h(t)$. Moreover, this curve is seen to accurately reproduce the original experimental profile, including even the small irregularities in the latter.
[1] G. I. Barenblatt, in Nonlinear Dynamics and Turbulence, edited by G. I. Barenblatt, G. Iooss, and D. D. Joseph (Pitman, Boston, 1983), p. 48.

[2] D. L. Youngs, Physica D 12, 32 (1984).

[3] K. I. Read, Physica D 12, 45 (1984).

[4] D. L. Youngs, Laser Part. Beams 12, 725 (1994).

[5] G. Dimonte and M. Schneider, Phys. Rev. E 54, 3740 (1996).

[6] G. Dimonte and M. Schneider, Phys. Plasmas 4, 4347 (1997).

[7] J. D. Ramshaw, Phys. Rev. E 58, 5834 (1998).

[8] G. Dimonte, Phys. Plasmas 6, 2009 (1999).

[9] J. D. Ramshaw, Phys. Rev. E 61, 5339 (2000).

[10] R. Clift, J. R. Grace, and M. E. Weber, Bubbles, Drops, and Particles (Academic, New York, 1978).

[11] J. C. V. Hansom, P. A. Rosen, T. J. Goldack, K. Oades, P. Fieldhouse, N. Cowperthwaite, D. L. Youngs, N. Mawhinney, and A. J. Baxter, Laser Part. Beams 8, 51 (1990).

[12] U. Alon, J. Hecht, D. Ofer, and D. Shvarts, Phys. Rev. Lett. 74, 534 (1995).

[13] G. Dimonte, Phys. Plasmas 7, 2255 (2000).

[14] G. Dimonte and M. Schneider, Phys. Fluids 12, 304 (2000).

[15] J. D. Ramshaw, Proceedings of the Nuclear Explosives Code Developers Collaborations (NECDC) 2000, Oakland, CA, 2000, Lawrence Livermore National Laboratory Report No. UCRL-JC-139800 (unpublished).

[16] G. Dimonte and R. Tipton, Phys. Fluids (to be published).

[17] N. Freed, D. Ofer, D. Shvarts, and S. A. Orszag, Phys. Fluids A 3, 912 (1991).

[18] A. W. Cook and P. E. Dimotakis, J. Fluid Mech. 443, 69 (2001); 457, 410 (2002).
[19] G. Dimonte, D. L. Youngs, A. Dimits, S. Weber, M. Marinak, S. Wunsch, C. Garasi, A. Robinson, M. J. Andrews, P. Ramaprabhu, A. C. Calder, B. Fryxell, J. Biello, L. Dursi, P. MacNeice, K. Olson, P. Ricker, R. Rosner, F. Timmes, H. Tufo, Y.-N. Young, and M. Zingale, Phys. Fluids 16, 1668 (2004).

[20] G. Dimonte, Phys. Rev. E 69, 056305 (2004).

[21] G. B. Wallis, One-Dimensional Two-Phase Flow (McGraw Hill, New York, 1969).

[22] D. A. Drew and S. L. Passman, Theory of Multicomponent Fluids (Springer-Verlag, New York, 1999).

[23] J. D. Ramshaw, Am. J. Phys. 70, 508 (2002).

[24] D. L. Youngs, Physica D 37, 270 (1989).

[25] D. L. Youngs, Phys. Fluids A 3, 1312 (1991).

[26] Y. A. Kucherenko, S. I. Balabin, R. Cherret, and J. F. Haas, Laser Part. Beams 15, 25 (1997).

[27] G. B. Whitham, Linear and Nonlinear Waves (Wiley, New York, 1974).

[28] R. B. Bird, W. E. Stewart, and E. N. Lightfoot, Transport Phenomena (Wiley, New York, 1960).

[29] Y. Zhou, G. B. Zimmerman, and E. W. Burke, Phys. Rev. E 65, 056303 (2002).

[30] V. H. Ransom and J. D. Ramshaw, in Dynamics of Two-Phase Flows, edited by O. C. Jones and I. Michiyoshi (CRC Press, Boca Raton, FL, 1992).

[31] R. Peyret and T. D. Taylor, Computational Methods for Fluid Flow (Springer-Verlag, New York, 1983).

[32] C. A. J. Fletcher, Computational Techniques for Fluid Dynamics, 2nd ed. (Springer-Verlag, Berlin, 1991), Vol. II. 